\title{
Duration of a "Brown-Like" Phenotype of White Adipose Tissue Induced by the $\beta 3$ Agonist CL-316,243
}

\author{
Authors \\ Wojciech Danysz ${ }^{1}$, Kang Jinlai ${ }^{2}$, Fugang Li $^{2}$
}

\author{
Affiliations \\ 1 Merz Pharmaceuticals, Frankfurt, Germany \\ 2 HD Biosciences Ltd, Shanghai, China
}

Key words

WAT, BAT, browning, UCP-1, H\&E

received 25.06 .2018

accepted 27.07.2018

\section{Bibliography}

DOI https://doi.org/10.1055/a-0667-8744

Published online: 6.9.2018

Drug Res 2019; 69: 265-270

(c) Georg Thieme Verlag KG Stuttgart · New York

ISSN 2194-9379

\author{
Correspondence \\ Wojciech Danysz \\ Merz Pharmaceuticals \\ Eckenheimer Landstrasse 100 \\ 60348 Frankfurt \\ Germany \\ Tel.: + 49/69/150 3564, Fax: + 49/69/15039564 \\ wojciech.danysz@merz.de
}

\begin{abstract}
"Browning" i. e. the transformation of white adipose tissue into brown-like adipose tissue could induce efficient burning of excess fat reserves via induction of non-shivering thermogenesis. For example, activation of $B 3$ adrenergic receptors has been show to induce such changes, however, it is still not clear, how long after termination of such a treatment, beneficial effects might be maintained. To address this question, we treated rats s.c. for 2 weeks with the ß3 agonist CL-316,243 at $1 \mathrm{mg} / \mathrm{kg}$ and assessed interscapular brown fat and inguinal white fat pads weight, UCP-1 (a marker for the brown-like fat phenotype) using immunohistochemistry and H\&E staining, at different intervals after treatment termination.

One 1 day after the treatment cessation there was a decrease of inguinal white fat pad weight and increase of interscapular fat pad. This change vanished at 7 days for inguinal pad and at 14 days for interscapular pad. Histological analysis of interscapular pads showed increased UCP-1 staining and brown-like morphology in $\mathrm{H} \& \mathrm{E}$ staining slices at 1 day, but not other time points. In case of inguinal pad there were brown-like features in H\&E slices at 1 day and less after 7 days, but absent at 14 days. UCP- 1 staining was only detected 1 day after the treatment.

In conclusion, the present results indicate that browning-like changes of white fat may be short lasting after treatment termination and could require maintenance treatment of inductor to achieve desired therapeutic effect. This might be a serious shortcoming of potential therapeutic use.
\end{abstract}

\section{Introduction}

Adipose tissue can be divided into the brown adipose tissue (BAT) type and white adipose tissue (WAT) type, both of which have different physiological functions. WAT is loaded with fat molecules and its main function is to store energy in such lipid droplets. Excess WAT leads to obesity, diabetes and other related diseases [1,2]. BAT is strongly expressed in children, but much less so in adults, and its role is to quickly burn energy to generate heat to support the body to adapt to the cold environment via non-shivering thermogenesis. The transformation of WAT into BAT has been suggested to be a very attractive treatment option for obesity and diabetes [1]. BAT activation resulting in increased "burning" of stored fat, heat production and a resultant decrease in fat depots has been observed in man in several clinical studies after exposure to cold
[3-5]. It has been reported that WAT in patients with severe burns gradually shifted to a BAT-like phenotype in both molecular and functional properties, suggesting that white fat in humans can undergo gradual "browning" to cope with such burn traumas and transform from an energy-storing to an energy-dissipating tissue [6]. Pharmacological treatments such as prednisolone or capsinoids under certain conditions activate BAT and increase energy expenditure in humans [2, 7-9]. Recently, WAT to BAT transformation after pharmacological treatment i. e. phosphodiesterase inhibitor Sildenafil has been demonstrated in man [10].

In animals, repetitive treatment with PPARy agonists, cannabinoid $\mathrm{CB} 1$ antagonists, or adenosine $\mathrm{A} 2 \mathrm{~A}$ receptor stimulation has been shown to increase UCP-1 in WAT, indicating a gain of BAT-like 
features [11-15]. Similar effects have been shown for activation of ß3 receptors by selective agonists such as CL-316,243 [12, 16-18].

However, it is still not known, how long such a brown fat-like phenotype in WAT adipocytes remains after termination of the treatment. This is crucial for therapeutic use since constant, long term treatment with e. g. some $B 3$ agonists may result in an increase in side-effects and loss of efficacy [19].

To address this issue we treated rats s.c. with CL-316,243, the activity of which we previously verified in our model [20], for 2 weeks at $1 \mathrm{mg} / \mathrm{kg}$ and assessed effects at different intervals after treatment termination. In such animals we assessed interscapular (BAT) and inguinal (WAT) fat pads weight and histology using UCP-1 and $\mathrm{H} \&$ E staining.

\section{Methods}

\section{Animals}

Male SD rats (200-300 g, Vital River Laboratories Animal Ltd.) were housed in Plexiglas cages ( 3 rats per cage) at a temperature $\left(22 \pm 3^{\circ} \mathrm{C}\right.$ ) and humidity ( $55 \pm 15 \%$ ) controlled rooms of an AAALAC accredited animal facility. Animals were provided with food (Keaoxieli certified Rodent Diet) and sterile water ad libitum and kept on a 12-h light/dark (6:00 am /6:00 pm) cycle acclimated for 1 week prior to the study.

\section{Reagents and consumables}

CL-316,243 disodium was purchased from Sequoia Research Products Ltd. (Pangbourne, UK) and dissolved in phosphate buffered saline with $\mathrm{pH}$ of 7.4. All other reagents were purchased from Sigma.

\section{Experimental design}

Rats were injected s.c. with sterile vehicle or CL-316,243 (1 mg/kg) daily for 14 days ( 4 groups, $N=8$ per group) as shown in $>$ Fig. $\mathbf{1}$. On sacrifice day, interscapular and inguinal fat pads were gently isolated, weighed and processed further for histological analysis (see below).

\section{Fat histology analysis}

Following completion of the experimental protocol, rats were deeply anesthetized and their fat pads (FP) were removed and placed in $10 \%$ neutral buffered formalin at room temperature for $24 \mathrm{~h}$. The
FP were then transferred to $15 \%$ sucrose in a phosphate buffer (PB, $0.1 \mathrm{M}, \mathrm{pH} 7.3$ ) solution at $4{ }^{\circ} \mathrm{C}$ for $24 \mathrm{~h}$, and finally placed in $30 \%$ sucrose solution in PB at $4{ }^{\circ} \mathrm{C}$ for $48 \mathrm{~h}$. After de-paraffinization /rehydration of tissue sections, the FP was histologically sectioned at $5 \mu \mathrm{m}$ using a cryostat and sections mounted directly onto gelatin coated slides. Heat mediated antigen retrieval conditioning in citrate buffer $(\mathrm{pH} 6)$ was performed on tissue sections before commencing with a standard IHC staining protocol. Briefly, slides were immerse in $3 \%$ hydrogen peroxide solution at room temperature for $15 \mathrm{~min}$ and next given a 20 min incubation in protein block, serum-free (Dako, Cat \#X0909). Afterwards, a further incubation for $1 \mathrm{~h}$ at RT in rabbit anti-UCP1 antibody (cat \#ab10983, Abcam) was applied at 1:500 dilution in antibody diluent (Dako, Cat \#S2022). Slides were then exposed to three 10 min washes in PB and incubated for 30 min in HRP Labelled polymer anti-rabbit (Dako, Cat \#K4003) followed by another three 10 min washes with PB. The distribution of peroxidase was revealed by incubating the sections in a DAB solution (Dako, Cat\#K8002) for 5 min before being counterstained with hematoxylin. Slides were allowed to dry and coverslipped with neutral balsam and sealed. Positive IHC area was quantified by IPP 6.0 software. The whole slide was scanned and all the positive area was selected with the same magnification. The pen tool was used to circle the positive area and view the statistics.

\section{Statistical analysis}

Results were expressed as mean \pm SEM or medians in case of nonGaussian distribution. Statistical analysis was performed using Student t-test or ANOVA on ranks followed by Man-Whitney test. The difference was considered significant when $p<0.05$.

\section{Results}

\section{Body weight}

There was no significant difference of body weight between CL316,243 and vehicle treated rats on the last day of treatment i.e. the values were: $389.4 \pm 8.5,387.5 \pm 5.1,387.5 \pm 8.2$ and $394.4 \pm 6.1 \mathrm{~g}$. for saline, and the three ( $\triangleright$ Fig. 1) CL-316,243 treated groups respectively (mean \pm SEM).

\section{Inguinal and interscapular fat pad weight/volume}

After 2 weeks of treatment with CL-316,243 there was a significant decrease in inguinal fat pad (IFP) weight one day (group 2) after

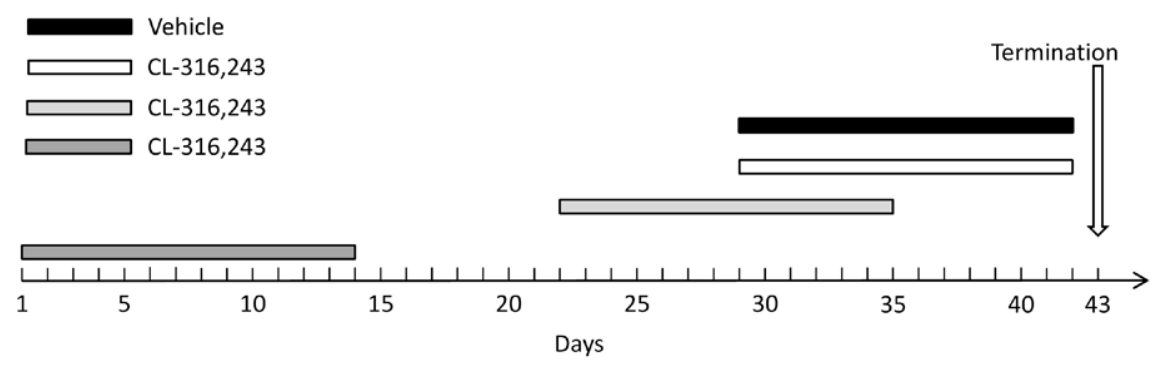

-Fig. 1 Schematic presentation of four treatment groups. Treatment of all three groups treated with CL-316,243 started at different time point in order to have termination for all groups on the same day. 
treatment termination ( $\triangleright$ Fig. 1; One way ANOVA). This effect vanished on day 7 and 14. Interestingly, an increase in interscapular fat pad weight was observed on day 1 and 7 (but not 14) after treatment termination ( $\triangleright$ Fig. 2).

\section{Fat pads histology analysis}

In animals treated with CL-316,243 there were signs of BAT activation in interscapular fat pad seen in $\mathrm{H} \&$ E slices and also increase in UCP-1 staining 1 day, but not 7 or 14 days after treatment termination (• Fig. 3).

In inguinal fat pad, treatment with CL-316,243 increased BAT like multiocular lipid droplets in inguinal fat as seen in slices stained with $\mathrm{H} \& \mathrm{E} 1$ day after treatment termination, less evident at 7 days and absent after 14 days (> Fig. 4).

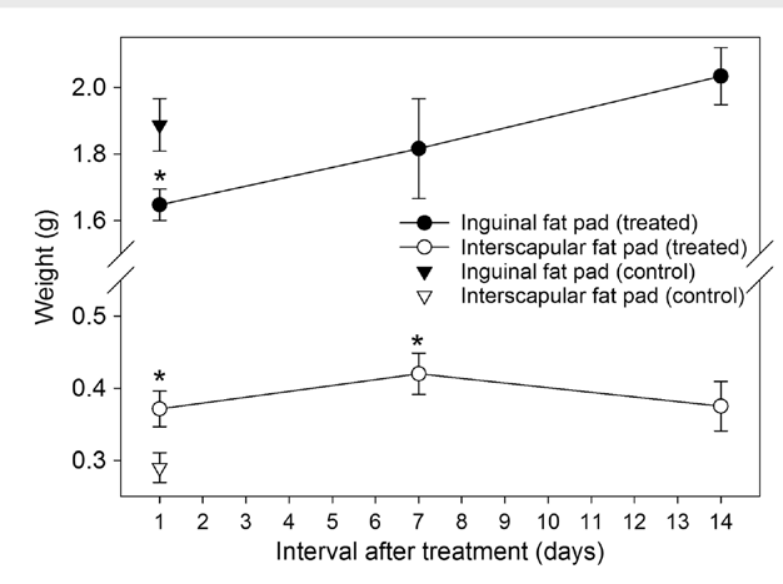

- Fig. 2 Effect of two weeks treatment with CL-316,243 on inguinal and interscapular fat pad weight 1,7 and 14 days after treatment termination. Results are expressed as mean \pm SEM. ${ }^{*}-\mathrm{P}<0.05$ vs. respective control (day 1 ) Student t-test. $N=8$.
CL-316,243 also increased staining for UCP-1 staining in inguinal fat pad suggesting transformation into BAT-like phenotype one day after treatment but not at other tested intervals. Respective median values were 1.1 ( $\%$ of UCP-1 positive cells) for 1 day interval and 0 for all other groups. The difference for 1 day was statistically significant (Kruskal Wallis ANOVA on ranks $\mathrm{H}=26.65, \mathrm{P}<0.001$ followed by Mann-Whitney test, $U=0.0, p<0.001)$. It should be noted that in two animals in 7 days group there was increase in UCP-1 observed (> Fig. $\mathbf{5}$ ).

\section{Discussion}

The present study showed that systemic treatment with CL316,243 for 2 weeks ( $1 \mathrm{mg} / \mathrm{kg}$ ) produced changes in WAT (inguinal fat pad) indicative transformation into a BAT-like phenotype or showing of beige like features such as characteristic adipocytes morphology and UCP-1 staining. Moreover, signs of native BAT activation were also obtained. This is in line with published literature on CL-316,243 showing after chronic treatment hypertrophy of BAT and BAT-like changes of WAT in rats on a fatty diet [21] as well as in mice [22]. Also, at the histological level, browning-like changes were observed in WAT of diabetic Zucker rats after similar treatment [23].

In addition to changes at the histological level, we also observed a decrease in inguinal fat pad weight indicating that enhanced energy expenditure may have taken place. Such a decrease in WAT pads weight has been previously described after chronic treatment with CL-316,243 in rats [21] and in mice [22].

Interestingly, the degree of cold-induced browning of WAT in various body sites of mice seems to correlate with sympathetic innervation [24] which suggests the role of B3 receptors as one of the major mediators. Therefore, data obtained in the present study with the $ß 3$ agonist may have some implication to other treatments/inductors producing browning. The formation of brown-like adipocytes in reaction to chronic cold stress in mice is reversed

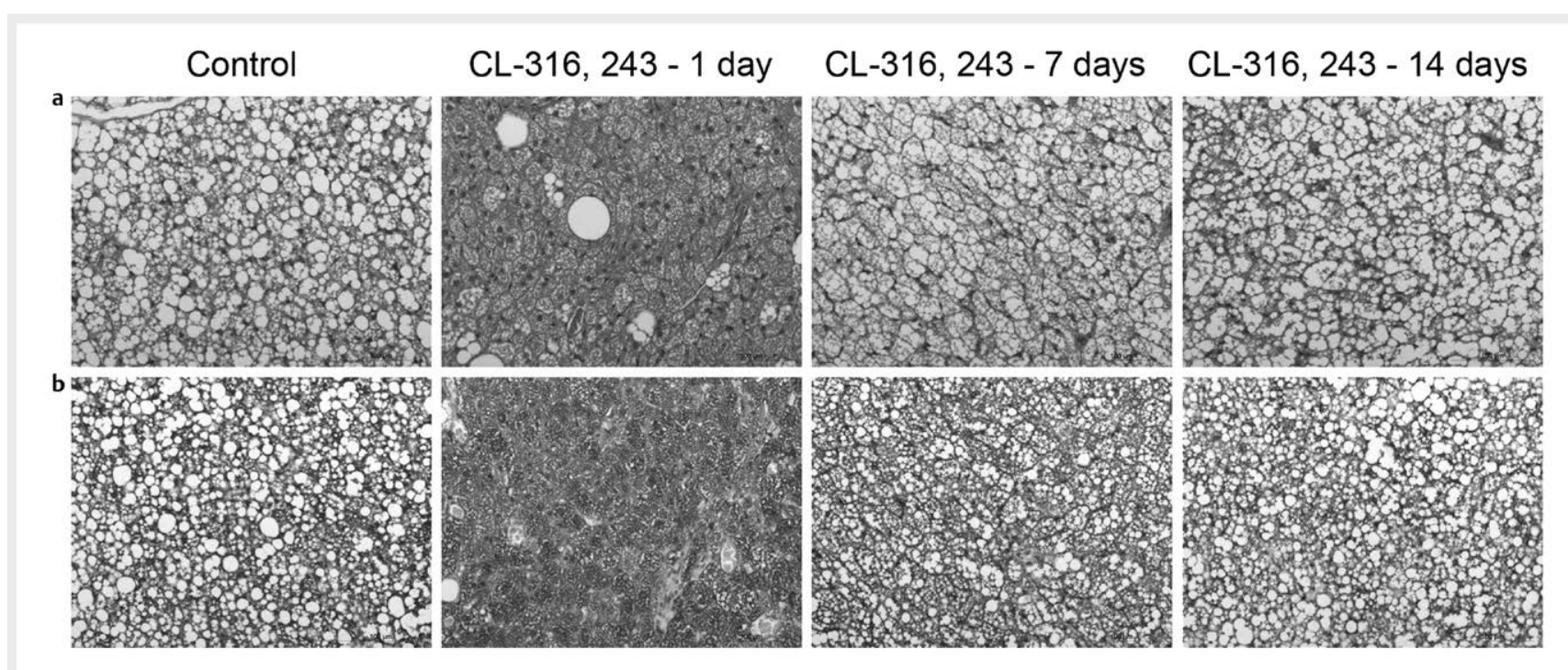

- Fig. 3 Effect of two weeks treatment with CL-316,243 on interscapular fat pad histology visualized as H\&E staining a and UCP-1 staining b 1, 7 and 14 days after treatment termination. Representative pictures from each treatment group are shown. 


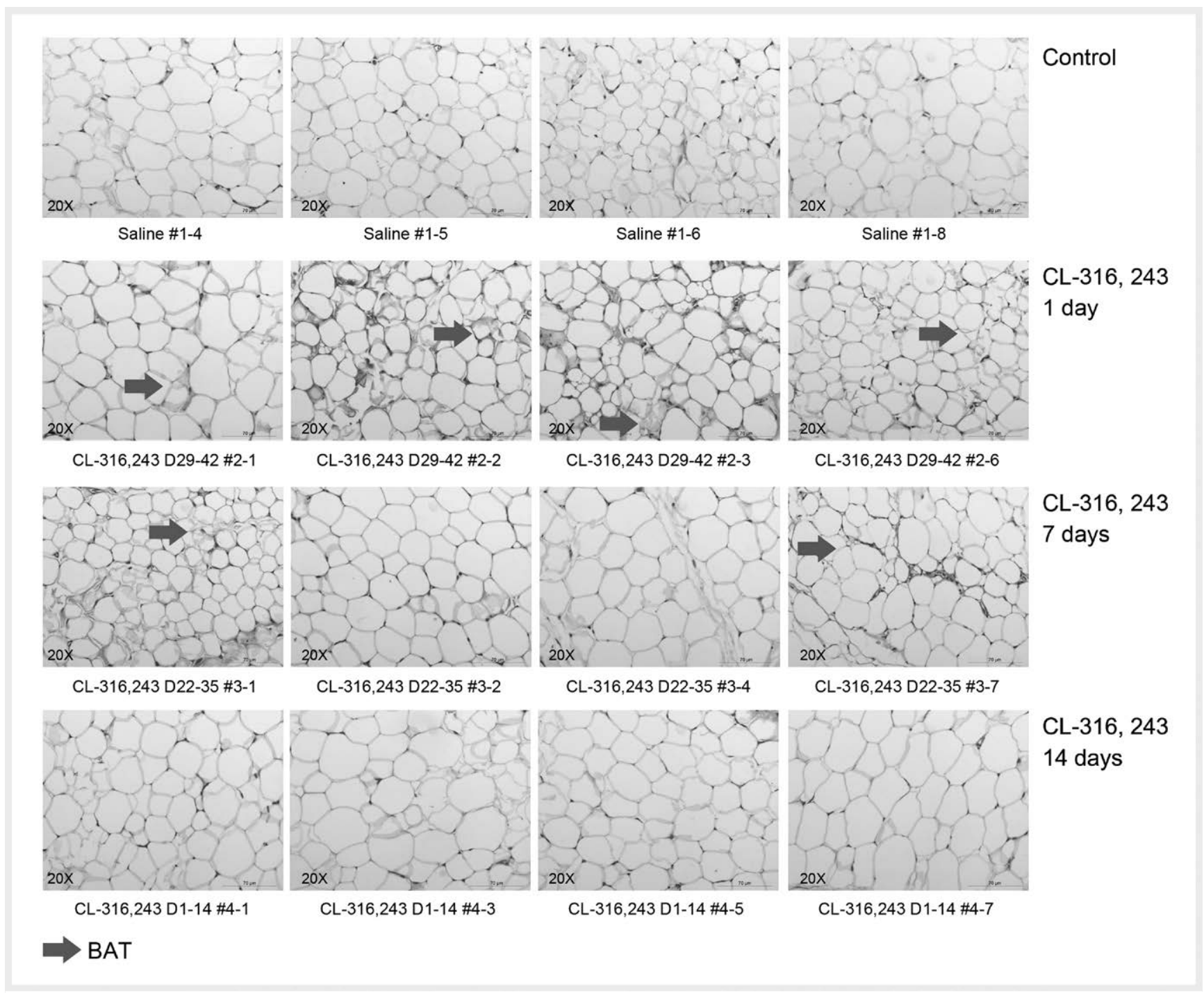

- Fig. 4 Effect of two weeks systemic s.c. injections of CL-316,243 on inguinal fat pad histology visualized as H\&E staining 1, 7 and 14 days after treatment termination. Four representative pictures from each treatment group are shown. Red arrows point to cells with BAT-like phenotype.

within 5 weeks of warm adaptation [25]. This is in concert with the present data suggesting that WAT to BAT-like transformation seems to be rather short lasting as it disappeared 7 or 14 days after the treatment had been stopped. Parallel to that observation, in native BAT (interscapular fat pad) activation was observed accompanied by an increase in weight one day after treatment termination but was absent 7 or 14 days later.

This may indicate some limitations of browning induction by pharmacological treatment for therapeutic effect as it may be short lasting and may require continues treatment. However, such long term treatment may have some limitations since for some pharmacological approaches tolerance to the therapeutic effects and significant increase of side-effects have been observed [19]. It should, however, be considered that this conclusion may not apply to all types of treatment (here specifically ß3 agonist CL-316,243) and not to all species (including man). It does however point to such a risk potential and it adds to other pitfalls connected with concept of "browning" of WAT in man e. g. it has so far been shown in man mainly after severe "traumatic events" like extensive skin burns or cachexia $[6,26]$.

\section{Conclusions}

The present study shows that the in vivo conversion of the WAT to BAT-like phenotype is short lasting and requires maintenance treatment with the activator (here $B 3$ agonist). This would be an important shortcoming of clinical application of WAT to BAT-like transformation, however species differences and specificity of type of the pharmacological target should be also taken into account.

\section{Acknowledgement}

Proofreading by Christopher G. Parsons is highly appreciated. 


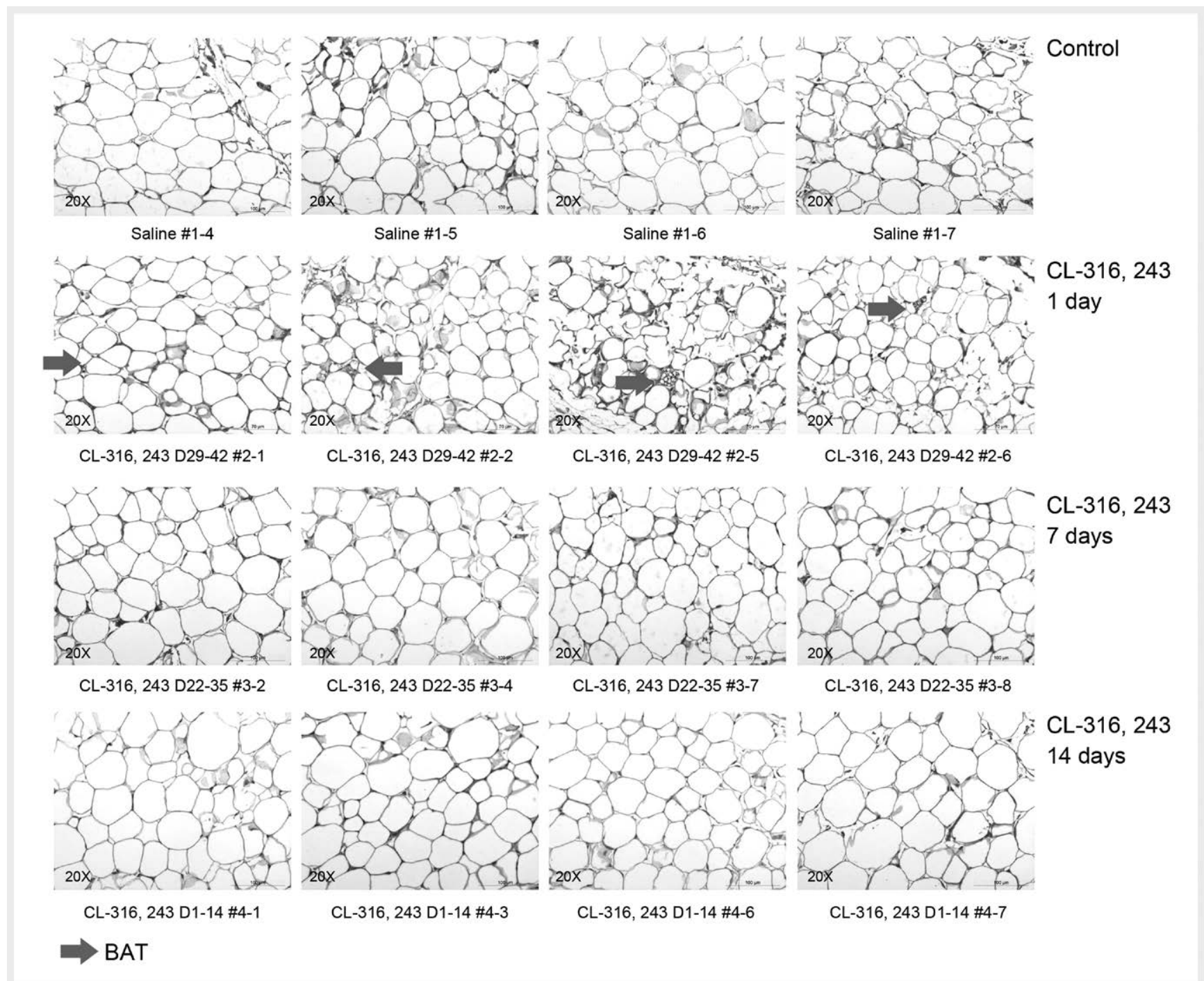

- Fig. 5 Effect of two weeks treatment with CL-316,243 on inguinal fat pad histology visualized as UCP-1 staining 1, 7 and 14 days after treatment termination. Four representative pictures from each treatment group are shown. Red arrows point to UCP-1 stained cells.

\section{Conflict of Interest}

The Authors have no conflict of interest to declare.

\section{References}

[1] Cannon B, Nedergaard J. Brown adipose tissue: Function and physiological significance. Physiol Rev 2004; 84: 277-359

[2] Yoneshiro T, Aita S, Matsushita M et al. Recruited brown adipose tissue as an antiobesity agent in humans. J Clin Invest 2013; 123: 3404-3408

[3] Orava J, Nuutila P, Lidell ME et al. Different metabolic responses of human brown adipose tissue to activation by cold and insulin. Cell Metab 2011; 14: 272-279

[4] Muzik O, Mangner T], Leonard WR et al. 150 PET measurement of blood flow and oxygen consumption in cold-activated human brown fat. J Nucl Med 2013; 54: 523-531
[5] van der Lans AA, Hoeks J, Brans B et al. Cold acclimation recruits human brown fat and increases nonshivering thermogenesis. J Clin Invest 2013; 123: 3395-3403

[6] Sidossis LS, Porter C, Saraf MK et al. Browning of subcutaneous white adipose tissue in humans after severe adrenergic stress. Cell Metab 2015; 22: 219-227

[7] Ramage LE, Akyol M, Fletcher AM et al. Glucocorticoids acutely increase brown adipose tissue activity in humans, revealing speciesspecific differences in UCP-1 regulation. Cell Metab 2016; 24: 130-141

[8] Sugita J, Yoneshiro T, Hatano T et al. Grains of paradise (Aframomum melegueta) extract activates brown adipose tissue and increases whole-body energy expenditure in men. Br J Nutr 2013; 110: 733-738

[9] Whiting S, Derbyshire E, Tiwari BK. Capsaicinoids and capsinoids. A potential role for weight management? A systematic review of the evidence. Appetite 2012; 59: 341-348

[10] Li S, Li Y, Xiang L et al. Sildenafil induces browning of subcutaneous white adipose tissue in overweight adults. Metabolism 2018; 78: 106-117 
[11] Gnad T, Scheibler S, von Kugelgen I et al. Adenosine activates brown adipose tissue and recruits beige adipocytes via A2A receptors. Nature 2014; 516: 395-399

[12] Himms-Hagen J, Melnyk A, Zingaretti MC et al. Multilocular fat cells in WAT of CL-316243-treated rats derive directly from white adipocytes. Am J Physiol Cell Physiol 2000; 279: C670-C681

[13] Koh YJ, Park BH, Park JH et al. Activation of PPAR gamma induces profound multilocularization of adipocytes in adult mouse white adipose tissues. Exp Mol Med 2009; 41: 880-895

[14] Perwitz N, Wenzel J, Wagner I et al. Cannabinoid type 1 receptor blockade induces transdifferentiation towards a brown fat phenotype in white adipocytes. Diabetes Obes Metab 2010; 12: 158-166

[15] Boon MR, van den Berg SA, Wang Y et al. BMP7 activates brown adipose tissue and reduces diet-induced obesity only at subthermoneutrality. PLoS One 2013; 8: e74083

[16] Liu X, Perusse F, Bukowiecki L]. Mechanisms of the antidiabetic effects of the beta 3-adrenergic agonist CL-316243 in obese Zucker-ZDF rats. Am J Physiol 1998; 274: R1212-R1219

[17] Yoshida T, Umekawa T, Kumamoto K et al. beta 3-Adrenergic agonist induces a functionally active uncoupling protein in fat and slow-twitch muscle fibers. Am J Physiol 1998; 274: E469-E475

[18] Weyer C, Gautier JF, Danforth E Jr. Development of beta 3-adrenoceptor agonists for the treatment of obesity and diabetes-an update. Diabetes Metab 1999; 25: 11-21
[19] de Souza C], Burkey BF. Beta 3-adrenoceptor agonists as anti-diabetic and anti-obesity drugs in humans. Curr Pharm Des 2001; 7: 1433-1449

[20] Danysz W, Han Y, Li F et al. Browning of white adipose tissue induced by the $\beta 3$ agonist CL-316,243 after local and systemic treatment PK-PD relationship. Biochim Biophys Acta 2018; 1864: 2972-2982

[21] Himms-Hagen J, Cui ], Danforth E Jr. et al. Effect of CL-316,243, a thermogenic beta 3-agonist, on energy balance and brown and white adipose tissues in rats. Am J Physiol 1994; 266: R1371-R1382

[22] Nagase I, Yoshida T, Kumamoto K et al. Expression of uncoupling protein in skeletal muscle and white fat of obese mice treated with thermogenic beta 3-adrenergic agonist. J Clin Invest 1996; 97 : 2898-2904

[23] Ghorbani M, Himms-Hagen J. Appearance of brown adipocytes in white adipose tissue during CL 316,243-induced reversal of obesity and diabetes in Zucker fa/fa rats. Int J Obes Relat Metab Disord 1997; 21: 465-475

[24] Murano I, Barbatelli G, Giordano A et al. Noradrenergic parenchymal nerve fiber branching after cold acclimatisation correlates with brown adipocyte density in mouse adipose organ. J Anat 2009; 214: 171-178

[25] Rosenwald M, Perdikari A, Rulicke T et al. Bi-directional interconversion of brite and white adipocytes. Nat Cell Biol 2013; 15: 659-667

[26] Petruzzelli M, Schweiger M, Schreiber R et al. A switch from white to brown fat increases energy expenditure in cancer-associated cachexia. Cell Metab 2014; 20: 433-447 